\title{
Risk factors and predictors that influence SARS-Cov-2 IgG positivity
}

\section{A cross-sectional study of blood donors in Riyadh, Saudi Arabia}

\author{
Mohammed F. Alosaimi, MD, Abdulkarim Alhetheel, PhD, Khalid A. Aleisa, MD, Abdullah A. Altwerki, MD, \\ Njoud M. Alenezy, MD, Ebtisam M. Almutairi, MD, Leen O. Alothaim, MD, Abdul Manan A. Khalid, MD, \\ Khalid M. Alayed, MD, Mohammed A. Almazyad, MD, Turki A. BinMoammar, MD, Fahdah A. Alshobaili, MD, \\ Fatimah S. Al-Shahrani, MD, Sarah Alsubaie, MD, Rana M. Hasanato, MD.
}

\begin{abstract}

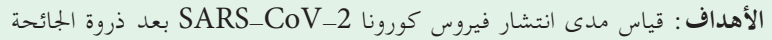

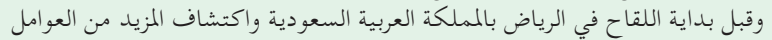

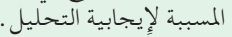

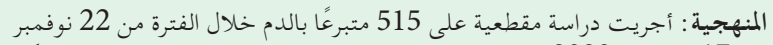

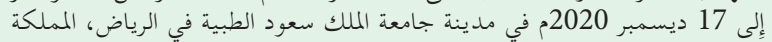

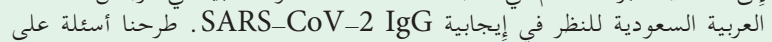

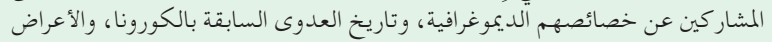
المرتبطة.

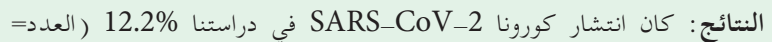

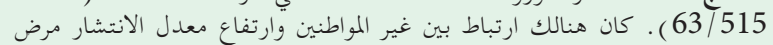

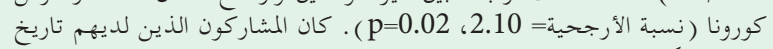

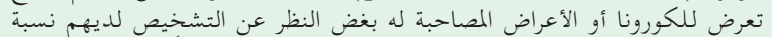

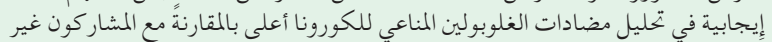

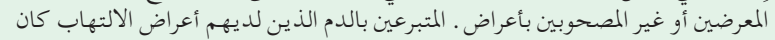

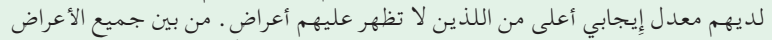

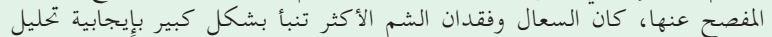

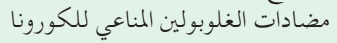

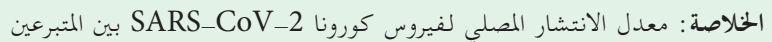

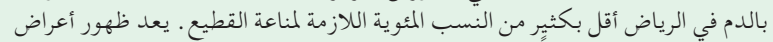
عARS_CoV-2
\end{abstract}

Objectives: To study the prevalence of severe acute respiratory syndrome coronavirus 2 (SARS-CoV-2) after pandemic's peak and before the vaccine enrollment in Riyadh, Saudi Arabia and further explore predictors for SARS-CoV-2 positivity.

Methods: A cross-sectional study of 515 blood donors from November 22 to December 17, 2020 was conducted at King Saud University Medical City, Riyadh, Saudi Arabia to look at SARS-CoV-2 immunoglobulin G (IgG) positivity. The participants were asked questions about their demographic characteristics, past SARS-CoV-2 infection, SARS-CoV-2related symptoms and exposures.

Results: The seroprevalence in our study was $12.2 \%$ $(n=63 / 515)$. Being a non-citizen was associated with significantly higher seroprevalence (OR 2.10, $p=0.02$ ). Participants with history of SARS-CoV-2 exposure or symptoms regardless of SARS-CoV-2 diagnosis had higher
SARS-CoV-2 IgG positivity compared to unexposed or asymptomatic participants (OR 2.47, $p=0.0008$ or $11.19, p=0.0001$, respectively). Blood donors who had symptomatic SARS-CoV-2 IgG infection had a higher SARS-CoV-2 IgG positivity rate (OR 5.04, $p=0.008$ ) and index value $(p=0.003)$ than the asymptomatic. Of all the reported symptoms, cough $(p=0.004)$ and anosmia $(p=0.002)$ were significant predictors of SARS-CoV-2 IgG.

Conclusion: The seroprevalence of SARS-CoV-2 among the blood donors in Riyadh, Saudi Arabia is considerably lower than the percentages necessary for herd immunity. Developing SARS-CoV-2-symptoms is the critical factor for higher seropositivity after SARS-CoV-2 exposure.

Keywords: COVID-19, SARS-CoV-2, blood donors, COVID-19 serological testing, seroepidemiologic studies, Saudi Arabia, anosmia

Saudi Med J 2021; Vol. 42 (8): 853-861 doi: 10.15537/smj.2021.42.8.20210238

From the Department of Pediatrics (Alosaimi, Almazyad), College of Medicine, King Saud University, Riyadh, Kingdom of Saudi Arabia; from the College of Medicine Research Center (Alosaimi), King Saud University, Riyadh, Kingdom of Saudi Arabia; from the Department of Pathology and Laboratory Medicine (Alhetheel, Hasanato), King Saud University, Riyadh, Kingdom of Saudi Arabia.; from the College of Medicine (Aleisa, Altwerki, Alenezy, Almutairi), King Saud University, Riyadh, Kingdom of Saudi Arabia; from the Blood Bank (Khalid), King Saud University, Riyadh, Kingdom of Saudi Arabia; from the Department of Medicine (Alayed), College of Medicine, King Saud University, Riyadh, Kingdom of Saudi Arabia; from the Department of Family and Community Medicine (BinMoammar, Alshobaili); from the Division of Infectious Disease, Department of Internal Medicine (Al-Shahrani), King Saud University, Riyadh, Kingdom of Saudi Arabia; and from the Pediatric Infectious Diseases Unit, Department of Pediatrics, (Alsubaie), College of Medicine, King Saud University, Riyadh, Kingdom of Saudi Arabia.

Received 31st March 2021. Accepted 15th June 2021.

Address correspondence and reprint request to: Dr. Mohammed F. Alosaimi, Department of Pediatrics, College of Medicine, King Saud University, Riyadh, Kingdom of Saudi Arabia. E-mail:malosaimi@ksu.edu.sa

ORCID: https://orcid.org/0000-0002-8025-3491 
S evere acute respiratory syndrome coronavirus- 2 (SARS-CoV-2) infection has severely impacted countries worldwide. It started in China, in late December 2019. ${ }^{1}$ As of February 21, 2021, the number of cases was over 110 million, and the number of deaths was around 2.4 million from 222 countries and territories according to the world health organization databas. $^{2}$

Severe acute respiratory syndrome coronavirus-2 infection appeared in Saudi Arabia on March 2, 2020, after which several preventative measures were launched, including partial and then total lockdown. ${ }^{3}$ Fortunately, due to the Saudi health authority's excellent response and efforts since the beginning of the pandemic, the country has had only one peak so far from June to August of 2020, and the lockdown was lifted on June 21, 2020. As of February 21, 2021, the number of confirmed SARS-CoV-2 cases in the Kingdom were 373,702 , and the deaths were 6,445 . Of the total cases, 63,312 were reported from the Riyadh region. ${ }^{4}$ A recent study that looked at all the confirmed cases of SARS-CoV-2 infection in Saudi Arabia from March 1, 2020 to June 20,2020, showed that majority of the infected patients were male $(71.7 \%)$, the median age was 36 , and only $64 \%$ were symptomatic. ${ }^{3}$ In addition to that, the reported incubation period is 6 days. $^{3}$

Severe acute respiratory syndrome coronavirus 2 infection presentation varies from no symptoms to severe acute respiratory distress syndrome and death. ${ }^{5}$ The cases are usually diagnosed by reverse transcriptasepolymerase chain reaction (RT-PCR). However, there is an unidentified proportion of cases in which people display mild or no symptoms or were never tested despite having symptoms. ${ }^{6,7}$ Hence, serological tests are important for providing better estimates of populationbased infection. ${ }^{8}$

Immune reaction to SARS-CoV-2 is diverse and critical for effective elimination. ${ }^{9}$ One of the late immune responses is the production of immunoglobulin by the adaptive immune system. ${ }^{10}$ Iummunoglobulin (Ig) M and $\operatorname{IgG}$ were produced at various time points during the SARS-CoV-2 infection. Particularly, IgG appears at the end of the first week of infection and can last for months and even years. ${ }^{11,12}$ As for SARS-CoV-2 IgG, several studies have demonstrated that by the 2nd or 3rd week after infection, most infected cases

Disclosure. Authors have no conflict of interests, and the work was not supported or funded by any drug company. have seroconverted. ${ }^{13-15}$ However, the extent to which these antibodies last is still understudied. Many studies have demonstrated that IgG peaked at around 1-2 months and lasted for up to $4-5$ months in a subset of patients. ${ }^{12,16-18}$ Patients with symptomatic SARS-CoV-2 infection have higher seropositivity than asymptomatic ones. ${ }^{11,19}$ Furthermore, the severity of the SARS-CoV-2 infection correlated with higher seropositivity. ${ }^{11}$ Despite serological tests' limitations with regard to the estimation of the prevalence of the SARS-CoV-2 pandemic, they can be the most significant tool in assessing the disease's spread if they are carried out frequently and serially.

Four SARS-CoV-2 IgG studies have been conducted in Saudi Arabia since the pandemic started. ${ }^{20-23}$ Three of the studies looked at the blood donors in the early phase of the pandemic (Jan-May, May, and May-June of 2020), ${ }^{21-23}$ and one looked at healthcare workers (HCWs) during May 2020. ${ }^{20}$ The results showed huge variability from $1.4 \%$ to $19.3 \%$ SARS-CoV-2 IgG positivity. ${ }^{20-23}$ In Riyadh, 2 studies looked at seroprevalence in May 2020 and found that the positivity was $0 \%$ in blood donors and $1.1 \%$ in HCW. ${ }^{20,23}$ However, none have evaluated SARS-CoV-2 seroprevalence after the peak and before the vaccine role out in the Kingdom to better understand the spread of the disease.

We aim in our study to look at the prevalence of SARS-CoV-2 infection in Saudi Arabia's capital 3 months after the pandemic peak and before the vaccine campaign started. Furthermore, we aim to explore past SARS-CoV-2 infection and exposure histories to predicts which factors might influence seropositivity.

Methods. A literature searches was conducted to find prior related research articles. We searched the following terminologies in PubMed (MEDLINE) using MeSH database: "SARS-CoV-2", "COVID-19 serological testing", "COVID-19", "Saudi Arabia", "seroconversion", "SARS-CoV-2 IgG", "SARS-CoV-2 IgM", "blood donors", "anosmia", and "Seroepidemiologic studies". We used these terminologies alone or in multiple combinations. The resulted articles were screen by authors for relevance and articles related to topics were chosen and cited.

This is a cross-sectional study to look at seroprevalence of SARS-CoV-2 among blood donors from the blood bank at King Saud University Medical City (KSUMC), Riyadh, Saudi Arabia from November 22 to December 17, 2020. This study was approved by the Local Institutional Review Board (KSU Ref. No. 20/0584/ IRB), and written informed consent was obtained. The study was conducted according to principles of Helsinki Declaration. 
As there was a lack of SARS-CoV-2 infection prevalence studies in Saudi Arabia before we conducted our study, an estimation of between $10-30 \%$ prevalence guided our sample size calculation. The sample size needed to estimate the study's prevalence was found to be between 139 and 323 based on a 5\% margin of error and 95\% confidence interval (CI). Provided the uncertainty of our estimation, we aimed to recruit approximately 500 subjects. At the end of our study, we were able to recruit 515 participants.

The inclusion criteria was based on the eligibility of blood donors who came to the blood bank at KSU. The blood bank eligibility criteria follows the American Association of Blood Banks (AABB) ${ }^{24}$ and Saudi Central Board for Accreditation of Healthcare Institutions (CBAHI) guidelines. ${ }^{25}$ Briefly, donors are required to be in good health, weigh more than $50 \mathrm{~kg}$, not use any of the prohibited medications, and be free from any condition that may pose a risk of harm to themselves or the recipients of their blood products. Additional requirements were introduced in light of the SARS-CoV-2 pandemic, which include no history of SARS-CoV-2 diagnosis, related symptoms, or exposure in the 30 days before donation. On the day of donation, their vital signs and blood hemoglobin levels are checked, and they must fill out a health questionnaire. A qualified person interviews them regarding their medical history, travel history, personal history, medication usage, past medical treatments, lifestyle, and life events. Once blood donors were deemed eligible, informed consent was obtained, and they filled out the study questionnaire. Donors were excluded if they did not sign an inform consent.

Covariates. The study questionnaire included 3 sections: Socio-demographic questions, past SARS-CoV-2 exposure, and past SARS-CoV-2 diagnosis. The socio-demographic variables included age, gender, city of residence and district, occupation, and nationality. SARS-CoV-2 exposure-related questions confirmed SARS-CoV-2 exposure and its timing. Severe acute respiratory syndrome coronavirus- 2 diagnosis-related questions established the diagnosis by rRT-PCR, the timing of the diagnosis, and SARS-CoV-2 symptoms including: fever, cough, shortness of breath (SOB), anosmia, headache, and diarrhea. Based on selfreported symptoms, participants were categorized as asymptomatic or symptomatic (having any symptoms).

Serological test. We took 3-5 milliliters of blood in yellow-top tubes (BD Vacutainer ${ }^{\bullet}$ blood collection, $\mathrm{BD}$, New Jersey, USA) from all participants who consented to the study during the donation process. To detect SARS-CoV-2 IgG, we used the Abbott Architect
SARS-CoV-2 IgG serological kit (Abbott Laboratories, Lake Forest, IL, USA) and the ARCHITECT i2000SR system (Abbott Laboratories, Lake Forest, IL, USA). The test was carried out within 24 hours of sample collection for all participants. The kit is a chemiluminescent microparticle immunoassay (CMIA)-based assay and it is approved by the United States and Saudi Arabia's food and drug administrations for clinical use. It has a reported sensitivity of $100 \%$ after 16-21 days of symptoms and $99.6-99.9 \%$ specificity. ${ }^{14,26,27}$

Statistical analysis. The confidence interval for the overall seroprevalence was calculated by a binomial test. We calculated odds raito, 95\% CI, and Chi-square $p$-value when comparing 2 percentages of 2 or more categorical variables. We employed a 2-tailed independent t-test to compare the means of 2 continuous variables. We used one-way ANOVA to compare the means of the SARS-CoV-2 IgG index value across 3 and more variables. A logistic regression model was adopted to look at the association between the sociodemographic variables and SARS-CoV-2 IgG positivity. We used all sociodemographic and exposure variables for this model. To predict which symptoms were associated with seropositivity, we used another logistic regression model including all SARS-CoV-2 symptoms accounting for age and gender. Diagnosis and exposure to SARS-CoV-2 were excluded from this logistic regression model; it was expected to be the primary source for antibody generation and the expected strong correlation with the SARS-CoV-2 symptoms. We used JASP (version 0.14.1) (Computer software, Amsterdam, the Netherlands) to conduct statistical analysis.

Results. A total of 515 blood donors participated in this study, which was conducted approximately 3 months after the pandemic's national and local (Riyadh) incident peak (Figure 1). The study was concluded just before the SARS-COV-2 vaccine campaign started (Figure 1). The majority of the participants were male $(84.1 \%$ ) (Table 1). Their mean age was 30.65 (minimum 18 , maximum 62, and SD 9,76). They were mostly Saudi (84.7\%) (Table 1). Most participants live in Riyadh $(96.3 \%)$ and they are representative of 5 geographical areas of Riyadh (Table 1). Only 13.8\% are working in the healthcare system. Of the 515 participants, 145 had a history of exposure to SARS-COV-2 (28.2\%), and 61 (11.8\%) had confirmed SARS-CoV-2 infection.

The seroprevalence of SARS-CoV-2 among the blood donors was $12.2 \%$. Males had a $13.4 \%$ seroprevalence, while females had 6.10\% ( $p=0.07)$ (Table 2). Looking at our cohort, we found out that 92 participants had 
Table 1 - General characteristics of blood donors.

\begin{tabular}{|c|c|c|}
\hline Characteristics & $\mathrm{n}$ & $(\%)$ \\
\hline \multicolumn{3}{|l|}{ Age } \\
\hline $18-29$ & 275 & $(53.4)$ \\
\hline $30-49$ & 213 & (41.4) \\
\hline $50-69$ & 27 & $(5.3)$ \\
\hline \multicolumn{3}{|l|}{ Gender } \\
\hline Male & 433 & $(84.1)$ \\
\hline Female & 82 & $(15.9)$ \\
\hline \multicolumn{3}{|l|}{ City of residence } \\
\hline Riyadh & 496 & $(96.3)$ \\
\hline Other & 19 & (3.7) \\
\hline \multicolumn{3}{|c|}{$\begin{array}{l}\text { Geographical area in } \\
\text { Riyadh }\end{array}$} \\
\hline Center & 59 & (11.5) \\
\hline North & 84 & (16.3) \\
\hline South & 55 & (10.7) \\
\hline East & 91 & $(17.7)$ \\
\hline West & 109 & $(21.2)$ \\
\hline \multicolumn{3}{|l|}{ Nationality } \\
\hline Saudi & 436 & $(84.7)$ \\
\hline Non-Saudi & 79 & (15.3) \\
\hline \multicolumn{3}{|l|}{ Occupation } \\
\hline $\mathrm{HCW}$ & 71 & (13.8) \\
\hline Non-HCW & 407 & $(79.0)$ \\
\hline \multicolumn{3}{|c|}{$\begin{array}{l}\text { Exposure to SARS- } \\
\text { CoV-2 }\end{array}$} \\
\hline Yes & 145 & (28.2) \\
\hline No & 370 & $(71.8)$ \\
\hline $\begin{array}{l}\text { Valu } \\
\text { percenta }\end{array}$ & $\begin{array}{l}\text { as nun } \\
\text { healt }\end{array}$ & norker \\
\hline
\end{tabular}

either a positive rRT-PCR in the past or a positive SARS-CoV-2 IgG. Hence, the total prevalence of SARS-CoV-2 infection was $17.7 \%(\mathrm{n}=92 / 515 ; 95 \%$ CI: $14.7-21.5 \%)$. If we exclude the participants with past SARS-CoV-2 infection, the seroprevalence drops to $6.8 \%$. There was no significant difference in seroprevalence between Riyadh residents and outside residents (Table 2). Furthermore, no statistically significant differences in seroprevalence were found among residents within Riyadh's 5 geographical areas (Table 2). The highest and lowest seroprevalences were found in donors who live in the south (16.4\%) and the center $(8.7 \%)$ of Riyadh, Saudi Arabia. However, non-citizens had a higher seroprevalence compared to citizens $(p=0.02)$ (Table 2). There was no statistically significant difference in seroprevalence between HCWs and non-HCWs (Table 2). Participants who had a history of exposure to SARS-CoV-2 had higher seroprevalence than participants who did not $(p=0.0008)$. Seroprevalence was significantly higher among those who have had SARS-COV-2 compared to those who had not $(p<0.0001)$ (Table 2$)$. Blood donors who had any symptoms suggestive of SARS-CoV-2, regardless of
Table 2 - Percentages of severe acute respiratory syndrome coronavirus-2 (SARS-Cov-2) immunoglobulin $\mathrm{G}$ positivity by demographic and exposure variables.

\begin{tabular}{|c|c|c|c|}
\hline Variables & Seroprevalence & OR $(95 \% \mathrm{CI})$ & $P$-value \\
\hline Overall & $63 / 515(12.23)$ & & \\
\hline \multicolumn{4}{|l|}{ Age } \\
\hline $18-29$ & $27 / 220(9.82)$ & 1 & 0.17 \\
\hline $30-49$ & $31 / 213(14.55)$ & $1.21(0.69 ; 2.19)$ & 0.48 \\
\hline $50-69$ & $5 / 18(18.52)$ & $2.74(0.90 ; 8.31)$ & 0.07 \\
\hline \multicolumn{4}{|l|}{ Gender } \\
\hline Male & $58 / 433(13.4)$ & $2.3(0.92 ; 6.13)$ & 0.07 \\
\hline Female & $5 / 82(6.10)$ & $0.42(0.16 ; 1.08)$ & 0.07 \\
\hline \multicolumn{4}{|l|}{ City of residence } \\
\hline Riyadh & $61 / 496(12.3)$ & $1.19(0.26 ; 5.28)$ & 0.81 \\
\hline Other & $2 / 19(10.53)$ & & 0.81 \\
\hline \multicolumn{4}{|l|}{$\begin{array}{l}\text { Geographical } \\
\text { area in Riyadh }\end{array}$} \\
\hline Center & $5 / 59(8.47)$ & 1 & 0.71 \\
\hline North & $10 / 84(11.90)$ & $1.28(0.41 ; 3.96)$ & 0.51 \\
\hline South & $9 / 55(16.36)$ & $2.11(0.66 ; 6.75)$ & 0.20 \\
\hline East & $10 / 91(10.99)$ & $1.33(0.43 ; 4.11)$ & 0.61 \\
\hline West & $11 / 109(10.09)$ & $1.21(0.40 ; 3.67)$ & 0.73 \\
\hline \multicolumn{4}{|l|}{ Nationality } \\
\hline Saudi & $47 / 436(10.78)$ & $0.47(0.25 ; 0.89)$ & 0.02 \\
\hline Non-Saudi & $16 / 79(20.25)$ & $2.10(1.12 ; 3.9)$ & 0.02 \\
\hline \multicolumn{4}{|l|}{ Occupation } \\
\hline $\mathrm{HCW}$ & $5 / 71(7.04)$ & $0.48(0.18 ; 1.25)$ & 0.14 \\
\hline Non-HCW & $55 / 407(13.51)$ & $2.06(0.79 ; 5.34)$ & 0.14 \\
\hline \multicolumn{4}{|l|}{$\begin{array}{l}\text { Exposure to } \\
\text { SARS-Cov-2 }\end{array}$} \\
\hline Yes & $31 / 145(21.38)$ & $2.47(1.46 ; 4.2)$ & 0.0008 \\
\hline No & $32 / 370(8.64)$ & $0.34(0.20 ; 0.59)$ & 0.0008 \\
\hline \multicolumn{4}{|l|}{$\begin{array}{l}\text { SARS-Cov-2 } \\
\text { infection }\end{array}$} \\
\hline Yes & $32 / 61(52.46)$ & $15.05(8.09 ; 28.01)$ & $<0.001$ \\
\hline No & $31 / 454(6.83)$ & $0.06(0.03 ; 012)$ & $<0.001$ \\
\hline \multicolumn{4}{|l|}{$\begin{array}{l}\text { Symptoms of } \\
\text { SARS-Cov-2 }\end{array}$} \\
\hline Yes & $29 / 61(47.54)$ & $11.19(6.07 ; 20.64)$ & $<0.001$ \\
\hline No & $34 / 454(7.49)$ & $0.08(0.048 ; 0.16)$ & $<0.001$ \\
\hline
\end{tabular}

whether they had a confirmed SARS-COV-2 diagnosis or not, had higher seroprevalence than asymptomatic donors did $(p<0.0001)$ (Table 1). Accounting for the socio-demographic and exposure variables, the multivariate logistic regression model emphasized the significance of exposure to SARS-CoV-2 and, to a lesser extent, non-citizens with regard to SARS-CoV-2 IgG positivity (Table 3 ).

As our study was conducted 3 months after the pandemic's peak, we were able to look at the association between the timing of SARS-CoV-2 diagnosis and exposure and the rate of SARS-CoV-2 IgG positivity. We did not find a significant difference between the various time points after SARS-COV-2 diagnosis or 
exposure and SARS-CoV-2 IgG positivity or index values (Figure 2). Interestingly, the participants who had symptomatic SARS-CoV-2 infection had a much higher rate of SARS-CoV-2 IgG positivity (64.3\% vs. $26.3 \%, p=0.005)$ and higher mean index values compared to asymptomatic ones (means 2.7 vs. 1.00, $p=0.004$ ) (Figure 2). Using a multivariate logistical regression model, we found that cough or anosmia are statistically significant predictors of SARS-CoV-2 IgG positivity (Table 4). Among the donors who had exposure to SARS-COV-2, those having any symptoms suggestive of SARS-CoV-2 infection, regardless of whether they had confirmed SARS-CoV-2 infection or not, had significantly higher SARS-CoV-2 IgG positivity than asymptomatic donors did $(n=24 / 39$ $(61.5 \%)$ vs. $\mathrm{n}=7 / 106(6.6 \%)$, OR 22.63, $p<0.0001)$

Table 3 - Demographic and exposure predictors of SARS-CoV-2 antibody positivity.

\begin{tabular}{lccc}
\hline Variables & OR & 95\% CI & $P$-value \\
\hline Age & 1.031 & $0.99 ; 1.06$ & 0.068 \\
Gender (Male) & 1.683 & $0.55 ; 5.15$ & 0.362 \\
Nationality (non-Saudi) & 2.319 & $0.99 ; 5.38$ & 0.05 \\
Occupation (non-HCW) & 3.502 & $0.95 ; 12.84$ & 0.059 \\
Region center (West) & 1.403 & $0.41 ; 4.73$ & 0.586 \\
Region center (East) & 1.029 & $0.28 ; 3.69$ & 0.965 \\
Region center (South) & 2.408 & $0.66 ; 8.71$ & 0.18 \\
Region center (North) & 1.998 & $0.56 ; 7.06$ & 0.283 \\
Exposure to COVID19 (Yes) & 4.409 & $2.17 ; 8.92$ & $<0.001$ \\
\hline P-value were calculated using logistic regression model. OR: odds ratio, \\
\multicolumn{4}{c}{ CI: confidence interval, HCW: healthcare worker } \\
\hline \multicolumn{4}{c}{}
\end{tabular}

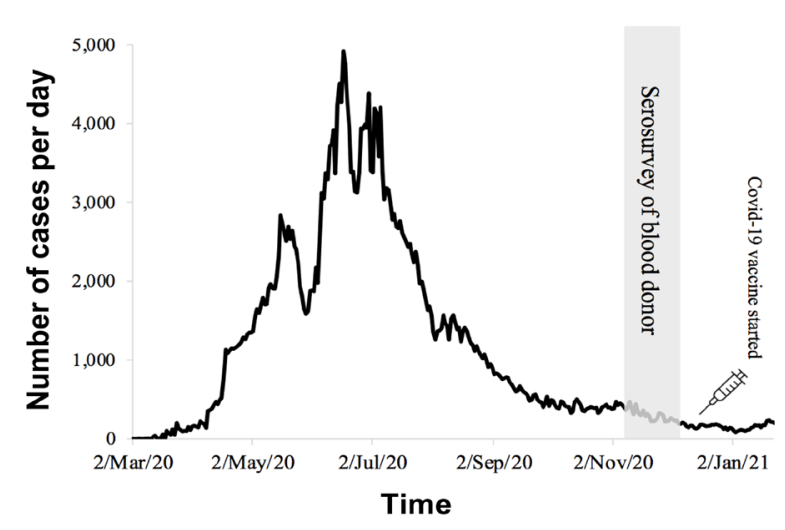

Figure 1 - Epidemic timeline of severe acute respiratory syndrome coronavirus-2 positive cases in Saudi Arabia. Gray area reflects the timing of enrollment to the study. Pfizer-BioNTech vaccines were introduce on December 17, 2020 in Saudi Arabia. Data from Saudi Ministry of Health, Covid-19 Command and Control Center CCC (https://covid19.moh. gov.sa).
Table 4 - Clinical symptoms predictors of severe acute respiratory syndrome coronavirus- 2 antibody positivity.

\begin{tabular}{lccc}
\hline Symptoms & OR & $95 \% \mathrm{CI}$ & $P$-value \\
\hline Fever (Yes) & 2.199 & $0.73 ; 6.61$ & 0.161 \\
SOB (Yes) & 1.177 & $0.27 ; 5.13$ & 0.828 \\
Anosmia (Yes) & 5.795 & $1.90 ; 17.62$ & 0.002 \\
Diarrhea (Yes) & 0.977 & $0.22 ; 4.30$ & 0.976 \\
Headache (Yes) & 1.303 & $0.36 ; 4.69$ & 0.686 \\
Cough (Yes) & 6.592 & $1.82 ; 23.86$ & 0.004 \\
\hline
\end{tabular}

$P$-value were calculated using logistic regression model. OR: odds ratio, CI: confidence interval, SOB; shortness of breath

(Figure 2). Lastly, comparing asymptomatic controls (no history of past SARS-CoV-2 infection or exposure) with asymptomatic patients exposed to and infected by SARS-CoV-2, we found that additional SARS-CoV-2 exposure had not changed the seropositivity. Only confirmed SARS-CoV-2 infection led to a significant difference between the asymptomatic groups (Figure 2).

Discussion. Seroprevalence studies are indispensable for detecting the magnitude of a pandemic and monitoring it. Such studies are especially important when a large percentage of individuals affected by a disease are asymptomatic, like the case of SARS-CoV-2 infection. In this cross-sectional study, we looked at the SARS-CoV-2 seropositivity rate among blood donors in the city of Riyadh 3 months after the peak and examined risk factors and predictors for SARS-CoV-2 IgG positivity. For the 515 participants, the seropositivity was $12.2 \%$, and the overall cumulative prevalence of SARS-CoV-2 infection was $17.9 \%$. These percentages are much higher than the incidence rate reported from the positive rRT-PCR data in Riyadh $(0.8 \%){ }^{4}$ However, our result is similar to that reported in the Madina region, Saudi Arabia during June 2020 (19.3\% seroprevalence). ${ }^{21}$ Notably, the Madina region has the highest incidence rate in Saudi Arabia $(1.3 \%),{ }^{4}$ and this is likely why they reported higher seroprevalence than us even though their study was conducted during the early part of the pandemic's peak. The other study in Riyadh only looked at HCWs in May 2020 and found seroprevalence to be $1.06 \%{ }^{20}$ In our cohort, a large percentage of the participants had confirmed SARS-CoV-2 by rRT-PCR ( $\mathrm{n}=61 / 515,11.8 \%)$. This is much higher than the positive rRT-PCR cases in Riyadh reported by the health authority $(0.8 \%)$. This could inflate our study's seroprevalence and might not reflect Riyadh's true prevalence. If we exclude the participants with past SARS-CoV-2 infection, the seroprevalence drops to $6.8 \%$. Of the seropositive participants, $49.2 \%$ 
A

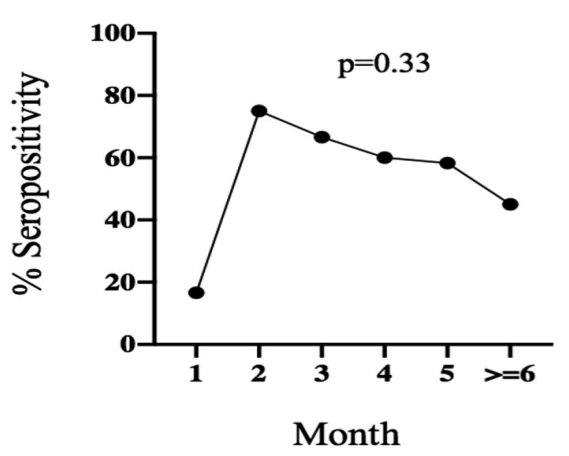

C

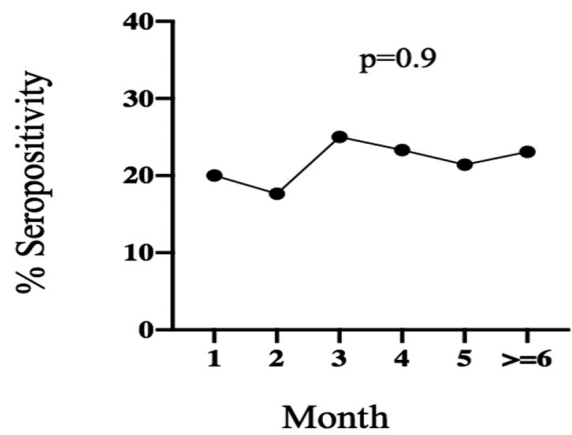

E

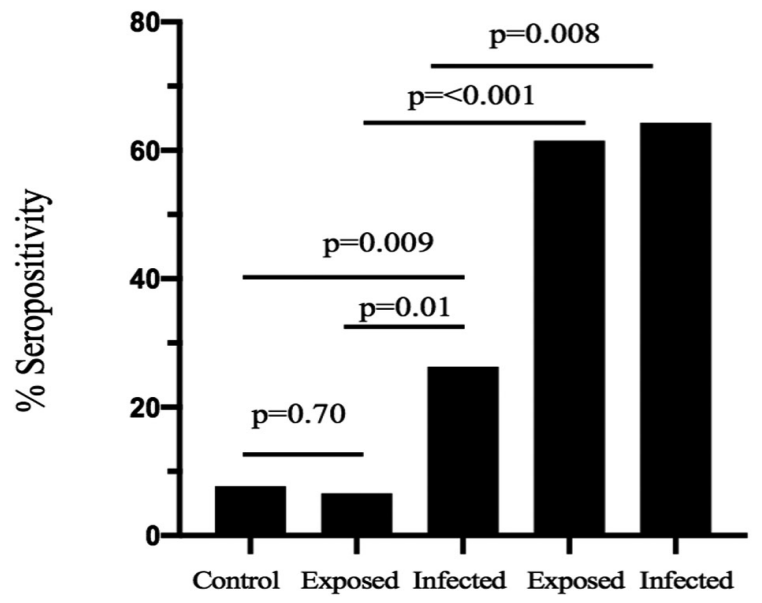

B

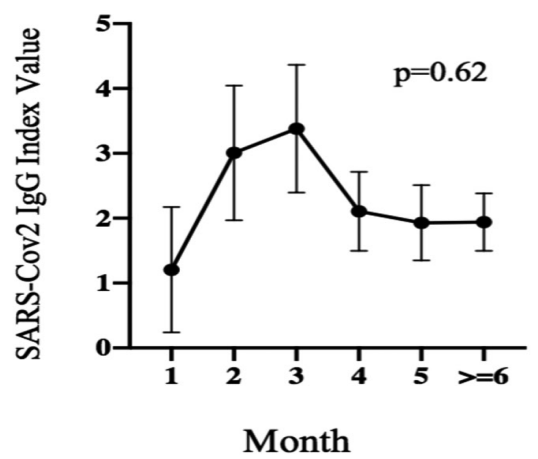

D

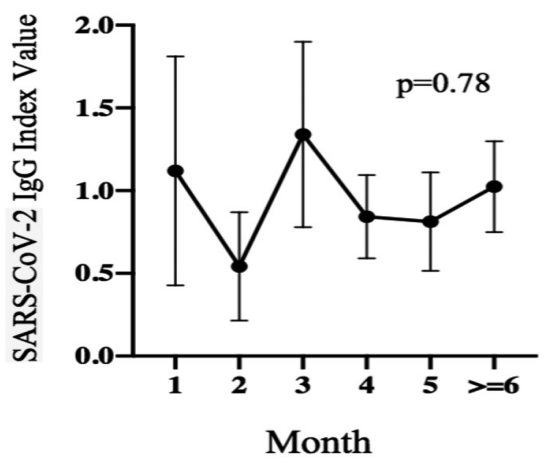

$\mathbf{F}$

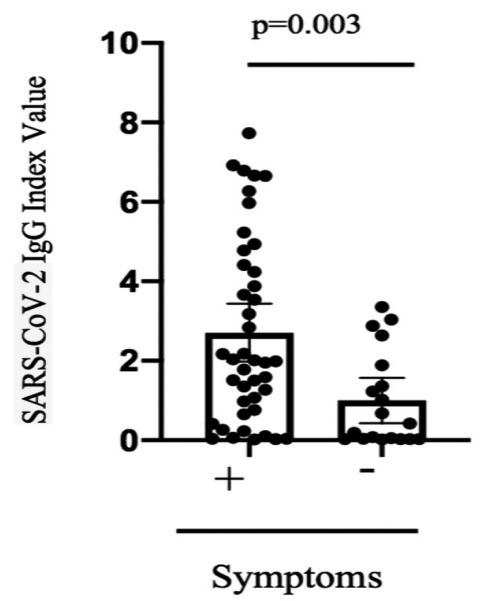

Figure 2 - Severe acute respiratory syndrome coronavirus-2 (SARS-CoV-2) related risk factors for seropositivity. A) Seropositivity percentages of different time points from participants who had SARS-CoV-2 disease. B) SARS-CoV-2 IgG index value across different time points from participants who had SARS-CoV-2 disease. C) Seropositivity percentages of different time points from participants who had SARS-CoV-2 exposure. D) SARS-CoV-2 IgG index value across different time points from participants who had SARS-CoV-2 exposure. E) Comparing seropositivity percentages of controls (non-exposed and non-infected) with participants who had exposure to SARS-CoV-2 or had been infected with it. F) SARS-CoV-2 IgG index of symptomatic vs. asymptomatic SARS-CoV-2 infected participants. Bars in E represent frequency and Chi-squared test were used, bars in F represents mean, and 95\% CI and t-test were used. 
had not previously been diagnosed with SARS-CoV-2, and $31.1 \%$ of participants who had SARS-CoV-2 infection were asymptomatic. This indicates that a large percentage of infections were undetected and emphasizes the importance of serological studies in estimating SARS-CoV-2 prevalence. These observations can be found in multiple SARS-CoV-2 seroprevalence studies. ${ }^{28-32}$

Our participants adequately represented the Riyadh area. Riyadh city is a geographically large and heavily populated city, which resulted in demographic differences in its areas that might affect the prevalence of SARS-CoV2infection. An example of these differences includes, but is not limited to, socioeconomic status and population density. However, in our analyses, there was no significant difference between different areas. Although the difference between genders was not statistically significant, males had higher seropositivity in our study, and this reflects the finding that most of the reported SARS-COV-2 cases affected males in Saudi Arabia $\left(71.7 \%\right.$ cases). ${ }^{3}$ Although HCWs have more SARS-COV-2 exposure risk, we found no statistical difference between HCWs and non-HCWs in terms of seroprevalence. Our results were similar to those of several studies and may reflect the role of adherence to personal protective measures in mitigating the risk of infection. ${ }^{33-35}$ However, we found that noncitizens had double the seroprevalence than citizens did. This is in line with the previous seroprevalence studies in Saudi Arabia conducted earlier during the pandemic, which also reported more SARS-COV-2 cases among non-citizens. ${ }^{23}$ Recently, a review of the clinical features of SARS-COV-2 infection in almost $64 \%$ of all reported cases in the Kingdom showed that non-citizens were infected more and had more severe presentations. ${ }^{3}$ Furthermore, Saudi Arabia has a high percentage of non-citizens who form a large proportion of the workforce in low-income jobs. They mostly live in crowded housing complexes, which could lead to higher exposure risk.

As we had a fair number of participants who either had SARS-CoV-2 exposure or symptoms or had confirmed infection by rRT-PCR, we were able to investigate these variables, identify factors associated with seropositivity, and predict it. Exposure to SARS-CoV-2 infection was positively associated with higher seropositivity, even if the exposure happened more than 6 months ago. This can be explained by the fact that exposure to SARS-CoV-2 increases the chances of our contracting SARS-CoV-2 infections and subsequently developing an immune response. Many previous studies have expressed similar observations. ${ }^{12,19,28,29}$ Strikingly, the development of any symptoms of SARS-CoV-2 after exposure was the determining factor for higher seropositivity. Participants who did not develop symptoms after exposure to SARS-CoV-2 had similar seroprevalence as non-exposed participants. Many factors could have contributed to this, including the type of exposure, duration of exposure, presence of personal protective equipment, or weak immune response in the asymptomatic group. A recent study found no significant difference in terms of Th 1 and Th 2 cytokine levels between the MERS-CoV asymptomatic group and the control group. ${ }^{36}$ Nonetheless, this interesting finding could mean that the individuals who did not develop symptoms after SARS-CoV-2 exposure were not infected or had lost SARS-CoV-2 IgG quickly.

In participants who had SARS-CoV-2 infection, being symptomatic resulted in significant seropositivity. This is likely because infected individuals who develop an infection do mount a more robust immune system. ${ }^{27}$ Multiple studies have shown that asymptomatic individuals lose their SARS-CoV-2 IgG quicker than symptomatic ones do. ${ }^{19,37,38}$ Furthermore, the SARS-CoV-2 IgG response increases with an increase in the severity score of the disease. ${ }^{39-41}$ Although we saw that the percentages and the mean value of SARS-CoV-2 IgG followed a logical pattern of initial increase after one month of infection and then a slow decline over 6 months, it was not statistically significant-likely due to the small sample size. SARS-CoV-2 infected individuals lose their SARS-CoV-2 IgG over time. This phenomenon has been observed with SARS-CoV-2 and other beta-coronaviruses. ${ }^{16,18,42}$ For instance, a followed-up study involving $9 \mathrm{HCW}$ sho survived MERS-CoV infection showed that 5 patients developed a MERS-CoV-IgG antibody within 3 months. However, after 15 months, the $\operatorname{IgG}$ antibodies were detected in only 2 patients. ${ }^{20}$ Another study in which 23 survivors of SARS-CoV infection were followed up for serologic testing for up to 6 years post-infection found a decline in SARS-CoV-IgG antibodies in all patients from the first month post-infection; these antibodies reached undetectable levels 4.5 years post-infection in 21 patients. ${ }^{43}$ Thus, it appears that the nature of infection with all beta-coronaviruses is marked by the induction of relatively weak and short-lived humoral immunity. This likely explains why almost half of our SARS-CoV-2 infected participants lost or did not have SARS-CoV-2 IgG.

Looking at the participants who reported a symptom compatible with SARS-CoV-2 infection, regardless of whether they had an rRT-PCR-confirmed infection or not, we found that having any symptoms of fever, 
cough, SOB, anosmia, diarrhea, or headache was associated with higher seropositivity. However, when we employed a logistical regression model, only cough and anosmia significantly predicted seropositivity. Anosmia has explicitly and strongly been associated with SARS-CoV-2 infection. Some reports have found similar results. ${ }^{4-47}$

Study limitaions. Our study limited by multiple factors, including a single center study which not representative of the general population; the study lacked participants belonging to the older population ( $>65$ years) and had skewed male representation and low rate of participation from non-citizen residents. Another limitation is that SARS-CoV-2 IgG was carried out in one-time point and might not reflect the true immunity to SARS-CoV-2. Finally, the response to the questionnaire likely is affected by participants' recall bias. Our study finding highlights the need for larger multiple center studies, longitudinal studies and follow-up studies to establish the disease extent clearly. Our study filled an important gap in our knowledge on the extent of the pandemic just before the vaccine started. It also adds an interesting finding that being asymptomatic after SARS-COV-2 exposure is not associated with increased seropositivity.

In conclusion, the seroprevalence we found is way below the required prevalence for herd immunity, which is $70-80 \% .{ }^{48}$ This indicates that society is still susceptible to this virus and that strict controlling measures have to be followed until we vaccinate enough people. Our study will encourage the local health authority to aggressively secure the SARS-COV-2 vaccine as it is the only safe and feasible option for containing this pandemic.

Acknowledgment. This work was supported by the College of Medicine Research Center, Deanship of Scientific Research, King Saud University, Riyadh, Kingdom of Saudi Arabia. We would like to thank PaperTrue (https://cn.papertrue.com/) for English language editing.

\section{References}

1. Zhu N, Zhang D, Wang W, Li X, Yang B, Song J, et al. A novel coronavirus from patients with pneumonia in China, 2019. $N$ Engl J Med 2020; 382: 727-733.

2. World Health Organizaiton. WHO Coronavirus Disease. [Updated 2021. Accessed 2021 Feb 21]. Available from: https:// covid19.who.int/

3. Alahmari AA, Khan AA, Elganainy A, Almohammadi EL, Hakawi AM, Assiri AM, et al. Epidemiological and clinical features of COVID-19 patients in Saudi Arabia. J Infect Public Health 2021; 14: 437-443.

4. Ministry of Health. COVID 19 Dashboard: Saudi Arabia. [Updated 2021. Accessed 2021 Feb 21]. Available from: https:// covid19.moh.gov.sa/
5. Wiersinga WJ, Rhodes A, Cheng AC, Peacock SJ, Prescott HC. Pathophysiology, transmission, diagnosis, and treatment of coronavirus disease 2019 (COVID-19): A review. JAMA 2020; 324: 782-793.

6. Mizumoto K, Kagaya K, Zarebski A, Chowell G. Estimating the asymptomatic proportion of coronavirus disease 2019 (COVID-19) cases on board the diamond princess cruise ship, Yokohama, Japan. Euro Surveill 2020; 25: 2000180.

7. Kimball A, Hatfield KM, Arons M, James A, Taylor J, Spicer $\mathrm{K}$, et al. Asymptomatic and presymptomatic SARS-CoV-2 infections in residents of a long-term care skilled nursing facility — King County, Washington, March 2020. MMWR Morb Mortal Wkly Rep 2020; 69: 377-381.

8. Public Health Considerations: Serologic Testing for COVID-19. [Updated 2020. Accessed 2021 February 22]. Available from: https://www.aphl.org/programs/preparedness/crisismanagement/documents/serologic-Testing-for-COVID-19.pdf

9. Vabret N, Britton GJ, Gruber C, Hegde S, Kim J, Kuksin M, et al. Immunology of COVID-19: current state of the science. Immunity 2020; 52: 910-941.

10. European Centre for Disease Prevention and Control. Immune responses and immunity to SARS-CoV-2. COVID19 latest Evid. (Updated 2020. Accessed June 2020]. Available from: https://www.ecdc.europa.eu/en/covid-19/latest-evidence/ immune-responses

11. Wang Y, Zhang L, Sang L, Ye F, Ruan S, Zhong B, et al. Kinetics of viral load and antibody response in relation to COVID-19 severity. J Clin Invest 2020; 130: 5235-5244.

12. Huang AT, Garcia-Carreras B, Hitchings MDT, Yang B, Katzelnick LC, Rattigan SM, et al. A systematic review of antibody mediated immunity to coronaviruses: kinetics, correlates of protection, and association with severity. Nat Commun 2020; 11: 4704.

13. Poustchi H, Darvishian M, Mohammadi Z, Shayanrad A, Delavari A, Bahadorimonfared A, et al. SARS-CoV-2 antibody seroprevalence in the general population and high-risk occupational groups across 18 cities in Iran: a population-based cross-sectional study. Lancet Infect Dis 2021; 21: 473-481.

14. Jääskeläinen AJ, Kuivanen S, Kekäläinen E, Ahava MJ, Loginov $\mathrm{R}$, Kallio-Kokko H, et al. Performance of six SARS-CoV-2 immunoassays in comparison with microneutralisation. J Clin Virol 2020; 129: 104512.

15. Ng DL, Goldgof GM, Shy BR, Levine AG, Balcerek J, Bapat SP, et al. SARS-CoV-2 seroprevalence and neutralizing activity in donor and patient blood. Nat Commun 2020; 11: 1-7.

16. Iyer AS, Jones FK, Nodoushani A, Kelly M, Becker M, Slater $\mathrm{D}$, et al. Persistence and decay of human antibody responses to the receptor binding domain of SARS-CoV-2 spike protein in COVID-19 patients. Sci Immunol 2020; 5: eabe0367.

17. Isho B, Abe KT, Zuo M, Jamal AJ, Rathod B, Wang JH, et al. Persistence of serum and saliva antibody responses to SARS-CoV-2 spike antigens in COVID-19 patients. Sci Immunol 2020; 5: eabe5511.

18. Jiang XL, Wang GL, Zhao XN, Yan FH, Yao L, Kou ZQ, et al. Lasting antibody and $\mathrm{T}$ cell responses to SARS-CoV-2 in COVID-19 patients three months after infection. Nat Commun 2021; $12: 897$.

19. Long QX, Tang XJ, Shi QL, Li Q, Deng HJ, Yuan J, et al. Clinical and immunological assessment of asymptomatic SARS-CoV-2 infections. Nat Med 2020; 26: 1200-1204. 
20. Alserehi HA, Alqunaibet AM, Al-Tawfiq JA, Alharbi NK, Alshukairi AN, Alanazi KH, et al. Seroprevalence of SARS-CoV-2 (COVID-19) among healthcare workers in Saudi Arabia: comparing case and control hospitals. Diagn Microbiol Infect Dis 2021; 99: 115273.

21. Mahallawi WH, Al-Zalabani AH. The seroprevalence of SARS-CoV-2 IgG antibodies among asymptomatic blood donors in Saudi Arabia. Saudi J Biol Sci 2020; 28: 1697-1701.

22. Alandijany TA, El-Kafrawy SA, Al-Ghamdi AA, Qashqari FS, Faizo AA, Tolah AM, et al. Lack of antibodies to SARS-CoV-2 among blood donors during COVID-19 lockdown: A study from Saudi Arabia. Healthcare (Basel) 2021; 9: 51.

23. Banjar A, Al-Tawfiq JA, Alruwaily A, Alserehi H, Al-Qunaibet A, Alaswad R, et al. Seroprevalence of antibodies to SARS-CoV-2 among blood donors in the early month of the pandemic in Saudi Arabia. Int J Infect Dis 2021; 104: 452-457.

24. Cells H. Eligibility determination for donors of human cells, tissues, and cellular and tissue-based products. Final rule. Fed Regist 2004; 69: 29785-29834.

25. Saudi Central Board for Accreditation Of Healthcare Institutions. National Standards for Clinical Laboratories \& blood banks. 1st ed. 2015; 5-7.

26. Bryan A, Pepper G, Wener MH, Fink SL, Morishima C, Chaudhary A, et al. Performance characteristics of the Abbott architect sars-cov-2 igg assay and seroprevalence in Boise, Idaho. J Clin Microbiol 2020; 58: e00941-20.

27. Abbott Laboratories. Abbott ARCHITECT IgG SARS-CoV-2 assay. [Update 2020. Assessed 2021 Febarury 22]. Available from: https://www.fda.gov/media/137383/download

28. Garcia-Basteiro AL, Moncunill G, Tortajada M, Vidal M, Guinovart C, Jiménez A, et al. Seroprevalence of antibodies against SARS-CoV-2 among health care workers in a large Spanish reference hospital. Nat Commun 2020; 11: 3500.

29. Lahner E, Dilaghi E, Prestigiacomo C, Alessio G, Marcellini L, Simmaco M, et al. Prevalence of Sars-Cov-2 infection in health workers (HWs) and diagnostic test performance: the experience of a teaching hospital in central Italy. Int J Environ Res Public Health 2020; 17: 1-12.

30. Behrens GMN, Cossmann A, Stankov MV, Witte T, Ernst D, Happle C, et al. Perceived versus proven SARS-CoV-2-specific immune responses in health-care professionals. Infection 2020; 48: 631-634.

31. Cento V, Colagrossi L, Nava A, Lamberti A, Senatore S, Travi $\mathrm{G}$, et al. Persistent positivity and fluctuations of SARS-CoV-2 RNA in clinically-recovered COVID-19 patients. J Infect 2020; 81: e90-e92.

32. Iversen $\mathrm{K}$, Bundgaard $\mathrm{H}$, Hasselbalch RB, Kristensen JH, Nielsen PB, Pries-Heje M, et al. Risk of COVID-19 in health-care workers in Denmark: an observational cohort study. Lancet Infect Dis 2020; 20: 1401-1408.

33. Amendola A, Tanzi E, Folgori L, Barcellini L, Bianchi S, Gori $\mathrm{M}$, et al. Low seroprevalence of SARS-CoV-2 infection among healthcare workers of the largest children hospital in Milan during the pandemic wave. Infect Control Hosp Epidemiol 2020; 41: 1468-1469.

34. Galanis P, Vraka I, Fragkou D, Bilali A, Kaitelidou D. Seroprevalence of SARS-CoV-2 antibodies and associated factors in healthcare workers: a systematic review and metaanalysis. J Hosp Infect 2020; 108: 120-134.
35. Piccoli L, Ferrari P, Piumatti G, Jovic S, Rodriguez BF, Mele F, et al. Risk assessment and seroprevalence of SARS-CoV-2 infection in healthcare workers of COVID-19 and nonCOVID-19 hospitals in Southern Switzerland. The Lancet Regional Health - Europe 2020; 1: 100013.

36. Alhetheel A, Albarrag A, Shakoor Z, Somily A, Barry M, Altalhi $\mathrm{H}$, et al. Assessment of Th1/Th2 cytokines among patients with Middle East respiratory syndrome coronavirus infection. Int Immunol 2020; 32: 799-804.

37. Bolk E, Matuschek C, Fischer J. Loss of anti-SARS-CoV-2 antibodies in mild Covid-19. N Engl J Med 2020; 383: 1694-1695.

38. Nag DS, Chaudhry R, Mishra M, Rai S, Gupta M. A prospective study on rapidly declining SARS-CoV-2 IgG antibodies within one to three months of testing IgG positive: Can it lead to potential reinfections? Cureus 2020; 12: e11845.

39. Marklund E, Leach S, Axelsson H, Nyström K, Norder H, Bemark M, et al. Serum-IgG responses to SARS-CoV-2 after mild and severe COVID-19 infection and analysis of IgG nonresponders. PLoS One 2020;15: e0241104.

40. Ren L, Zhang L, Chang D, Wang J, Hu Y, Chen H, et al. The kinetics of humoral response and its relationship with the disease severity in COVID-19. Commun Biol 2020; 3: 780.

41. Chen X, Pan Z, Yue S, Yu F, Zhang J, Yang Y, et al. Disease severity dictates SARS-CoV-2-specific neutralizing antibody responses in COVID-19. Signal Transduct Target Ther 2020; 5: 180.

42. Deeks JJ, Dinnes J, Takwoingi Y, Davenport C, Spijker R, Taylor-Phillips S, et al. Antibody tests for identification of current and past infection with SARS-CoV-2. Cochrane Database Syst Rev 2020; 6: CD013652.

43. Tang F, Quan Y, Xin Z-T, Wrammert J, Ma M-J, Lv H, et al. Lack of peripheral memory $B$ cell responses in recovered patients with severe acute respiratory syndrome: A six-year follow-up study. J Immunol 2011; 186: 7264-7268.

44. Tong JY, Wong A, Zhu D, Fastenberg JH, Tham T. The prevalence of olfactory and gustatory dysfunction in COVID-19 patients: A systematic review and meta-analysis. Otolaryngol Head Neck Surg 2020; 163: 3-11.

45. Wee LE, Chan YFZ, Teo NWY, Cherng BPZ, Thien SY, Wong $\mathrm{HM}$, et al. The role of self-reported olfactory and gustatory dysfunction as a screening criterion for suspected COVID-19. Eur Arch Otorhinolaryngol 2020; 277: 2389-2390.

46. Lechien JR, Chiesa-Estomba CM, De Siati DR, Horoi M, Le Bon SD, Rodriguez A, et al. Olfactory and gustatory dysfunctions as a clinical presentation of mild-to-moderate forms of the coronavirus disease (COVID-19): a multicenter European study. Eur Arch Otorhinolaryngol 2020; 277: 2251-2261.

47. Moein ST, Hashemian SM, Mansourafshar B, Khorram-Tousi A, Tabarsi P, Doty RL. Smell dysfunction: a biomarker for COVID-19. Int Forum Allergy Rhinol 2020; 10: 944-950.

48. Murray CJL, Piot P. The potential future of the COVID-19 pandemic: Will SARS-CoV-2 become a recurrent seasonal infection? JAMA 2021; 325: 1249-1250. 